\title{
Clinical Study Significance of Image Guidance to Clinical Outcomes for Localized Prostate Cancer
}

\author{
Qiuzi Zhong, Hong Gao, Gaofeng Li, Xia Xiu, Qinhong Wu, Ming Li, and Yonggang Xu \\ Department of Radiotherapy, Beijing Hospital, No. 1 Dongdan Dahua Road, Dongcheng District, Beijing 100730, China \\ Correspondence should be addressed to Gaofeng Li; lgf6243@163.com
}

Received 4 June 2014; Accepted 22 June 2014; Published 13 July 2014

Academic Editor: An Liu

Copyright (C) 2014 Qiuzi Zhong et al. This is an open access article distributed under the Creative Commons Attribution License, which permits unrestricted use, distribution, and reproduction in any medium, provided the original work is properly cited.

Purpose. To compare toxicity profiles and biochemical tumor control outcomes between patients treated with image-guided intensity-modulated radiotherapy (IG-IMRT) and non-IGRT intensity-modulated radiotherapy (IMRT) for clinically localized prostate cancer. Materials and Methods. Between 2009 and 2012, 65 patients with localized prostate cancer were treated with IGIMRT. This group of patients was retrospectively compared with a similar cohort of 62 patients who were treated between 2004 and 2009 with IMRT to the same dose without image guidance. Results. The median follow-up time was 4.8 years. The rectal volume receiving $\geq 40$ and $\geq 70$ Gy was significantly lower in the IG-IMRT group. Grade 2 and higher acute and late GI and GU toxicity rates were lower in IG-IMRT group, but there was no statistical difference. No significant improvement in biochemical control at 5 years was observed in two groups. In a Cox regression analysis identifying predictors for PSA relapse-free survival, only preradiotherapy PSA was significantly associated with biochemical control; IG-IMRT was not a statistically significant indicator. Conclusions. The use of image guidance in the radiation of prostate cancer at our institute did not show significant reduction in the rates of GI and GU toxicity and did not improve the biochemical control compared with IMRT.

\section{Introduction}

The practice of primary external-beam radiotherapy (EBRT) for prostate cancer has changed dramatically. There were many studies showing intensity-modulated radiotherapy (IMRT) that improved survival rates and reduced side effects comparing with conventional three-dimensional conformal radiation therapy (3D-CRT) for the treatment of prostate cancer $[1,2]$. With clinically proven benefit, IMRT became the standard care for prostate cancer. Image-guided radiotherapy (IGRT) uses 3D volumetric image to position the patient to treatment position before radiation delivery. IGRT potentially represents a more accurate form of dose delivery for patients receiving radiotherapy for prostate cancer. Given the highly conformal nature of this form of therapy, surrounding normal tissues such as the rectum and bladder can be effectively spared from being exposed to high radiation doses, leading to fewer treatment complications $[3,4]$. However, due to the longer treatment time and additional cost, it is important to understand the limitation of these new technologies and determine whether a dosimetric gain can translate into clinical benefit.
Since 2009, IG-IMRT has been the standard treatment at our institution for all prostate cancer patients. In this study, we retrospectively compared the outcomes of a cohort of patients treated with definitive IG-IMRT to the outcomes in a cohort treated with the same dose with IMRT but without image-guided position correction. The toxicity and tumorcontrol outcomes of these two patient cohorts were compared.

\section{Methods and Materials}

2.1. Patient Characteristics. From 2009 to 2012, 65 patients were treated with IG-IMRT to a dose of $76-80 \mathrm{~Gy}$ for stages T1-T3 prostate cancer. This group of patients was retrospectively compared with a similar cohort of 62 patients who were treated between 2004 and 2008 with IMRT to the same prescription dose, but without cone beam computed tomography (CBCT) guidance. The clinical characteristics of these two patient populations are shown in Table 1. Patients were staged according to the 2005 American Joint Committee on Cancer staging classification system. All patients had 
TABLE 1: Patient characteristic.

\begin{tabular}{|c|c|c|c|c|c|}
\hline \multirow[t]{2}{*}{ Patient demographics } & \multicolumn{2}{|c|}{$\begin{array}{l}\text { IG-IMRT } \\
(n=65)\end{array}$} & \multicolumn{2}{|c|}{$\begin{array}{l}\text { IMRT } \\
(n=62)\end{array}$} & \multirow[t]{2}{*}{$P$ value } \\
\hline & $n$ & $\%$ & $n$ & $\%$ & \\
\hline \multicolumn{6}{|l|}{ Preradiotherapy PSA } \\
\hline$<10$ & 58 & 89.23 & 49 & 79.03 & \multirow{3}{*}{0.286} \\
\hline $10-20$ & 4 & 6.15 & 7 & 11.29 & \\
\hline$>20$ & 3 & 4.62 & 6 & 9.68 & \\
\hline \multicolumn{6}{|l|}{ Total Gleason score } \\
\hline$<7$ & 10 & 15.38 & 12 & 19.35 & \multirow{3}{*}{0.559} \\
\hline 7 & 20 & 30.77 & 23 & 37.10 & \\
\hline$>7$ & 34 & 52.31 & 27 & 43.55 & \\
\hline \multicolumn{6}{|l|}{ T stage } \\
\hline $\mathrm{T} 1$ & 0 & 0 & 2 & 3.22 & \multirow{3}{*}{0.529} \\
\hline $\mathrm{T} 2$ & 30 & 46.15 & 27 & 43.55 & \\
\hline $\mathrm{T} 3$ & 35 & 53.85 & 33 & 53.23 & \\
\hline \multicolumn{6}{|l|}{ Age (y) } \\
\hline$<70$ & 14 & 21.54 & 18 & 29.03 & \multirow{2}{*}{0.331} \\
\hline$\geq 70$ & 51 & 78.46 & 44 & 70.97 & \\
\hline \multicolumn{6}{|l|}{ NCCN risk } \\
\hline Low & 2 & 3.08 & 2 & 3.23 & \multirow{3}{*}{0.933} \\
\hline Intermediate & 13 & 20.00 & 10 & 16.13 & \\
\hline High & 50 & 76.92 & 50 & 80.64 & \\
\hline \multicolumn{6}{|l|}{ Neoadjuvant ADT } \\
\hline Yes & 56 & 86.15 & 52 & 83.87 & \multirow{2}{*}{0.718} \\
\hline No & 9 & 13.85 & 10 & 16.13 & \\
\hline
\end{tabular}

ADT: androgen deprivation therapy; NCCN: National Comprehensive Cancer Network; PSA: prostate-specific antigen.

biopsy-proven adenocarcinoma that was classified according to the Gleason grading system, with neoadjuvant and concurrent androgen suppression therapy for prostate cancer.

For patients who received neoadjuvant androgen deprivation therapy (ADT; $n=108 ; 85.04 \%$ ), the median neoadjuvant ADT time was 8 months. The median preradiotherapy prostate-specific antigen (PSA) was $0.68 \mathrm{ng} / \mathrm{mL}$.

2.2. Target Definition. All patients underwent CT simulation approximately 2-4 days before starting treatment. A $5 \mathrm{~mm}$ slice thickness was used for CT image acquisition. The patients were instructed to have a full bladder and empty rectum at CT simulation and for each fraction of delivery.

Eclipse (Varian Medical Systems, Palo Alto, CA) was used for treatment planning. The treatment techniques, prescription dose, and dose-volume constraints for the target and normal tissues used for both patient groups were the same for the initial 66-70 Gy. For both groups, the planning target volume (PTV) included the prostate and entire seminal vesicles with a $8-10 \mathrm{~mm}$ margin except at the prostaterectal interface, where a $6 \mathrm{~mm}$ margin was used. For the IG-IMRT group, plan was modified for the last 5 fractions (10 Gy) with reduced PTV volume. In that boost plan, clinical target volume (CTV) was expanded with a three-dimensional margin of 3-4 mm to create a smaller PTV for planning.
The rationale at that time was not to deviate too much from our previous IMRT technique. But we hypothesized that the use of daily IGRT during the boost phase should justify reduced margin, given its enhanced positioning accuracy.

The pelvic lymph nodes were irradiated in patients with an estimated risk of pelvic nodal involvement of $\geq 15 \%$ using the Roach formula [5]. The pelvic nodal regions included the internal and external iliac, common iliac, perirectal and presacral nodal regions [6], and common iliac lymph nodes up to $\mathrm{L} 5 / \mathrm{S} 1$.

The bladder, rectum, small bowel, and femoral heads were delineated as OARs. The rectum volume included the entire rectal wall and the lumen, extending from the anus to the rectosigmoid flexure. The bladder was contoured from its base to the dome.

\subsection{Treatment Planning}

2.3.1. IMRT Group. Single-fraction dose of $2 \mathrm{~Gy}$ was prescribed to PTV. If CTV included pelvic nodal regions, the IMRT plan was given in two phases. In the first phase, the PTV1 which included pelvic lymphatic drainage area, seminal vesicles, and prostate received $48-50$ Gy in 24-25 fractions. Then a second CT simulation was performed. The PTV2 which only included prostate and seminal vesicles received 28-30 Gy boost in 14-15 fractions to a total PTV prostate dose of 76-80 Gy in 38-40 fractions. All doses were prescribed to a minimum isodose line encompassing $\geq 95 \%$ of the PTV. Patients were treated with 7 fields IMRT using Varian linear accelerators iX (Varian Medical Systems, Palo Alto, CA) equipped with a 120-leaf Varian millennium multileaf collimators. If CTV only included the prostate and entire seminal vesicles, the IMRT plan was finished in one phase: PTV received 76-78 Gy in 38-40 fractions. Doses to OARs were limited below thresholds. The dose constraints were rectum volume: $V_{70}<25 \%$ and $V_{50}<50 \%$; bladder: $V_{65}<$ $30 \%$ and $V_{50}<50 \%$; and femur: $V_{50}<5 \%$.

2.3.2. IG-IMRT Group. The prescription dose was the same with IMRT group. The only difference was in the last 5 fractions where a new plan was generated with reduced PTV margins.

2.4. Treatment Delivery and Outcomes Analyses. In the IGIMRT cohort, image guidance was achieved by using the onboard imaging (OBI) system on the Varian iX linear accelerator. Planning CT and the verification CBCT were registered first with automatic bony registration, followed by a manual registration based on the soft-tissue alignment and prostate position in the CBCT. No prostate-implanted markers were used. Daily imaging was performed during the first three fractions. Thereafter, IGRT was utilized one to two times weekly. For the last five fractions of treatment, CBCT was performed everyday. Please note that most of studies reported on prostate cancer using IGRT involved daily CBCT [7-9]. In our center, for the purpose of saving treatment time and minimizing additional radiation exposure, CBCT was only used 10-15 times through the entire course of treatment. The average number of CBCT was 12 times per patient. 
TABLE 2: Comparison of doses received by organs at risk between IMRT and IG-IMRT groups.

\begin{tabular}{lccc}
\hline & IG-IMRT & IMRT & $P$ \\
\hline Bladder & & & \\
$V_{40}(\%)$ & 57.88 & 58.32 & 0.937 \\
$V_{50}(\%)$ & 41.17 & 42.28 & 0.796 \\
$V_{60}(\%)$ & 27.48 & 30.62 & 0.905 \\
$V_{70}(\%)$ & 17.09 & 21.01 & 0.782 \\
Volume & 180.46 & 163.33 & 0.598 \\
Rectum & & & \\
$V_{40}(\%)$ & 53.358 & 61.286 & 0.022 \\
$V_{50}(\%)$ & 39.945 & 40.131 & 0.949 \\
$V_{60}(\%)$ & 29.316 & 26.500 & 0.210 \\
$V_{70}(\%)$ & 14.493 & 18.745 & 0.006 \\
Volume & 60.74 & 73.35 & 0.200 \\
\hline
\end{tabular}

$V_{40}, V_{50}, V_{60}$, and $V_{70}$ are the percentage of volume receiving $\geq 40, \geq 50, \geq 60$, and $\geq 70 \mathrm{~Gy}$, respectively. Data are presented as mean values.

Positioning errors were corrected online prior to treatment every time a CBCT was acquired.

Dose-volume histogram endpoints were retrospectively collected for all 127 patients. Using the composite plans, the bladder volume and rectum volume receiving $\geq 40\left(V_{40}\right), \geq 50$ $\left(V_{60}\right), \geq 60\left(V_{60}\right)$, and $\geq 70\left(V_{70}\right)$ Gy of the target volume were recorded. The assessment of toxicity was performed every week during treatment. Follow-up evaluations after RT were performed at intervals of 3 to 6 months, and the median follow-up time was 4.8 years (range, $1-8$ years). The median follow-up intervals for the IMRT and IG-IMRT cohorts were 70 and 23 months, respectively. PSA relapse was defined according to the Phoenix definition (absolute nadir plus $2 \mathrm{ng} / \mathrm{mL}$ dated at the call). All statistical analyses were performed using Statistical Package for Social Sciences, version 16.0 (SPSS, Chicago, IL). Gastrointestinal (GI) and genitourinary $(\mathrm{GU})$ toxicities were scored using the common terminology criteria for adverse events (CTCAE), version 3.0, grading schemes. Results were considered significant at a $P$ value $<0.05$.

\section{Results}

The patients' characteristics are summarized in Table 1. All patients completed RT, with no treatment breaks. The planning target volume dose coverage was not significantly different between IMRT and IG-IMRT for the prostate, seminal vesicles, and lymph nodes $(P>0.05)$.

3.1. Dosimetric Avoidance of Organs at Risk. Table 2 summarized the differences in dosimetry of the bladder and rectum for IMRT and IG-IMRT groups. The volume of bladder receiving $\geq 40, \geq 50, \geq 60$, and $\geq 70$ Gy was not significantly different from two groups $(P>0.05)$. The rectal volume receiving $\geq 40$ (61.3 versus $53.4 \mathrm{~Gy} ; P=0.022)$ and $\geq 70$ Gy (18.7 versus $14.5 \mathrm{~Gy} ; P=0.006)$ was significantly lower at the IGIMRT group.
TABLE 3: Comparison of acute rectal and urinary toxicities between patients treated with IG-IMRT and IMRT.

\begin{tabular}{lcccccc}
\hline Acute toxicities & \multicolumn{2}{c}{ IG-IMRT } & \multicolumn{2}{c}{ IMRT } & \multicolumn{2}{c}{ Total } \\
& $n=65)$ & \multicolumn{2}{c}{$(n=62)$} & \multicolumn{2}{c}{$(n=127)$} \\
Rectal & $n$ & $n$ & $\%$ & $n$ & $\%$ \\
0 & 47 & 72.3 & 45 & 72.6 & 92 & 72.4 \\
1 & 16 & 24.6 & 14 & 22.6 & 30 & 23.6 \\
2 & 2 & 3.1 & 3 & 4.8 & 5 & 4.0 \\
3 & 0 & 0.0 & 0 & 0.0 & 0 & 0.0 \\
Urinary & & & & & & \\
0 & 32 & 49.2 & 26 & 41.9 & 58 & 45.7 \\
1 & 23 & 35.4 & 24 & 38.7 & 47 & 37.0 \\
2 & 10 & 15.4 & 12 & 19.4 & 22 & 17.3 \\
3 & 0 & 0.0 & 0 & 0.0 & 0 & 0.0 \\
\hline
\end{tabular}

TABLE 4: Comparison of late rectal and urinary toxicities between patients treated with IG-IMRT and IMRT.

\begin{tabular}{lcccccc}
\hline Late toxicities & \multicolumn{2}{c}{$\begin{array}{c}\text { IG-IMRT } \\
(n=65)\end{array}$} & \multicolumn{2}{c}{$\begin{array}{c}\text { IMRT } \\
(n=62)\end{array}$} & \multicolumn{2}{c}{ Total } \\
& $n$ & $\%$ & $n$ & $\%$ & $n$ & $\%$ \\
\hline Rectal & & & & & & \\
0 & 60 & 92.3 & 57 & 92.0 & 117 & 92.1 \\
1 & 4 & 6.2 & 3 & 4.8 & 7 & 5.5 \\
2 & 1 & 1.5 & 1 & 1.6 & 2 & 1.6 \\
3 & 0 & 0.0 & 1 & 1.6 & 1 & 0.8 \\
Urinary & & & & & & \\
0 & 60 & 92.3 & 54 & 87.1 & 114 & 89.7 \\
1 & 4 & 6.2 & 6 & 9.7 & 10 & 7.9 \\
2 & 1 & 1.5 & 2 & 3.2 & 3 & 2.4 \\
3 & 0 & 0.0 & 0 & 0.0 & 0 & 0.0 \\
\hline
\end{tabular}

3.2. Acute Toxicity. Regarding the acute toxicity, 77 patients (67\%) experienced grade 1 symptoms and 27 (21\%) experienced grade 2 toxicity. No grade 3 or higher acute events were observed. The most common GU and GI radiation toxicities were frequency/urgency and diarrhea. Table 3 compared the incidence of acute rectal and urinary toxicities observed in the IG-IMRT and IMRT treatment groups. No differences were observed between the treatment groups for grade 2 and higher acute GI and GU toxicity rates $(P=0.986 ; P=0.6)$.

3.3. Late Toxicity. The grade 2 or higher late rectal toxicity was similar in and low for both the IGRT and non-IGRT groups (1.5\% and 3.2\%, resp.; $P=0.967)$. The most common late GI radiation effect was mild bleeding. One patient required minor cauterization for hemostasis. No late toxicity grade 4 was observed. The incidence of grade $0-1$ and 2 GU toxicity was $96.8 \%$ and $3.2 \%$, respectively, with IMRT compared with $98.5 \%$ and $1.5 \%$, respectively, with IG-IMRT (Table 4). In a logistic regression analysis, IGRT was not associated with significantly less acute and late toxicity compared with non-IGRT patients $(P>0.05)$. 


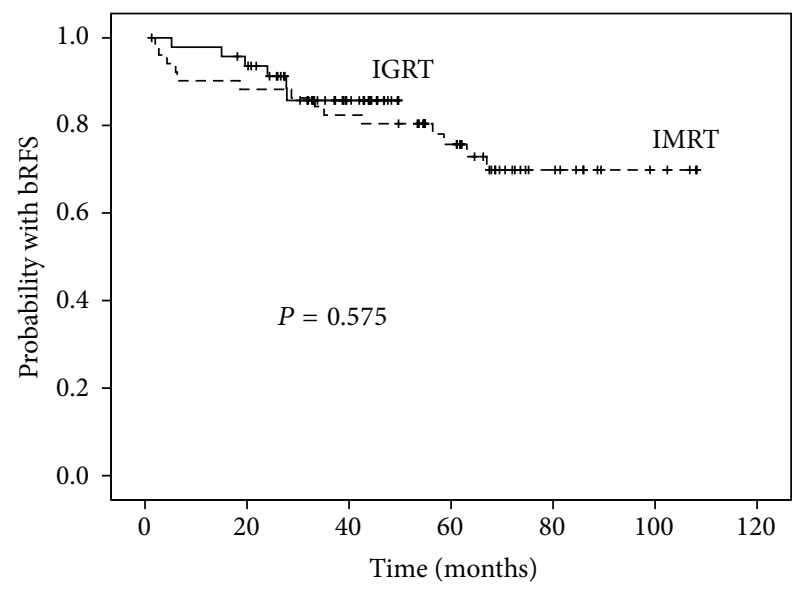

FIGURE 1: Comparison of prostate-specific antigen relapse-free survival outcomes between the high-risk patients treated with imageguided radiotherapy (IG-IMRT) and those treated with IMRT alone.

3.4. Biochemical Control. No significant improvement in biochemical control at 5 years was observed in the low- and intermediate-risk patients in IG-IMRT cohort compared with IMRT group $(P=0.427)$. As noted in Figure 1 , the same result was observed in the high-risk group patients $(P=0.575)$. In a Cox regression analysis identifying predictors for PSA relapse-free survival, only preradiotherapy PSA was significantly associated with biochemical control; image guidance was not (Table 5).

\section{Discussion}

Intuitively, IG-IMRT would provide enhanced delivery accuracy than IMRT alone. Thus IG-IMRT by default should demonstrate reduction in normal organ toxicity and better tumor-control outcomes. Chung et al. [10] have reported reduced acute bladder and rectal toxicities associated with whole pelvic IGRT compared with IMRT in prostate cancer patients. Their study used smaller PTV margins for IGIMRT group attributing it to the enhanced accuracy of image guidance. In 2009, de Crevoisier et al. described a series of 107 patients who underwent IG-IMRT (CBCT used in 67\% of patients, fiducial markers in $28 \%$, and ultrasounds in $5 \%$ ), with a median total dose of 76 Gy [11]. Grades 2 and 3 acute rectal toxicity rates were only $7 \%$ and $0 \%$, respectively. More recently, a retrospective comparison by Zelefsky et al. [12] noted that IGRT was associated with an improvement in biochemical tumor control among high-risk patients and a lower rate of late urinary toxicity compared with high-dose IMRT (86.4 Gy).

However, there are many factors that can potentially compromise the benefit of image guidance. For that reason, although numerous studies have demonstrated the dosimetric superiority of IG-IMRT compared with IMRT, very limited numbers of studies have shown that these dosimetric differences can lead to tangible differences to the patient in terms of toxicity and tumor control [12-14]. Our study
TABLE 5: Cox regression analysis for predictors identifying PSA relapse-free survival.

\begin{tabular}{lcccccc}
\hline Cox regression & \multirow{2}{*}{$B$} & SE & $P$ & $\operatorname{Exp}(B)$ & \multicolumn{2}{c}{$95 \%$ CI } \\
& & & & & Lower & Upper \\
\hline Gleason score $\geq 8$ & -0.120 & 0.480 & 0.802 & 0.887 & 0.346 & 2.273 \\
T stage & 0.419 & 0.460 & 0.362 & 1.520 & 0.617 & 3.744 \\
Age & 0.012 & 0.024 & 0.609 & 1.012 & 0.996 & 1.062 \\
Preradiotherapy PSA & 0.019 & 0.004 & 0.000 & 1.019 & 1.011 & 1.028 \\
IG & 0.609 & 0.546 & 0.265 & 1.838 & 0.630 & 5.362 \\
\hline
\end{tabular}

$B$ : regression coefficient estimate; SE: standard error; CI: confidence interval; IG: image guidance; PSA: prostate-specific antigen.

intended to show that without carefully cultivated implementation plan, addition of image guidance may not translate to clinically significant outcome improvements. In our study, both groups were treated to the same radiation dose level and with similar margins for the PTV. The patients' characters of two groups were almost identical $(P>0.05)$. Our results showed that there were no significant differences in side effects and clinical control between IG-IMRT and IMRT groups. Although these results are a little bit unexpected, they are not surprising. We believe the following factors could potentially compromise the benefit of image guidance.

(i) Frequency of Image Guidance. Most studies that demonstrated significant IGRT benefit used daily image guidance. Han et al. reported that daily image guidance was needed throughout the course of treatment in conformal radiotherapy for esophageal cancer. Even if the most frequent lessthan-daily IG strategy was to be used, substantial residual setup errors would occur for treatment fractions without image guidance, which could lead to significant daily dose variations for the target volume and adjacent normal tissues. However, one of the downsides of daily IGRT is the extra radiation dose it introduces [15-17]. Table 6 provides a list of the doses per fraction associated with a list of different IGRT modalities. There was at least one report stipulating that the extra imaging dose was associated with more toxicity if not computed in the total dose delivered [18]. Another consideration is the cost and resources. A randomized trial in France showed that the average increase of cost for CBCTbased daily imaging guidance was $43 \%$ higher than weekly imaging. They suggested that daily IGRT combined with intensity-modulated RT (IMRT) should only be considered in high-dose radiation delivery situations [19]. For the sake of reducing cost and imaging dose, we designed our study with less than daily image guidance. For each prostate patient treatment course, only 10-16 (mean $=12$ ) CBCTs were performed. For a treatment extending over a large number of fractions, the impact of day-to-day delivery variation, or random error, is usually less important than systematic errors [20-22]. However, when the number of fractions is reduced, then random error (amplified without image guidance) can have a larger negative impact [21]. Lack of daily CBCT in our study could be one of the reasons why the side effects of two groups were not significantly different. 
TABLE 6: Added dose and time per modality per fraction in pelvis IGRT [16, 50-53].

\begin{tabular}{lccc}
\hline Modality & Dose at midbody $(\mathrm{cGy})$ & ${\text { Time }(\mathrm{min})^{*}}^{\text {Available examples }^{\&}}$ \\
\hline Ultrasound & 0 & $2-3$ & BATCAM, Clarity \\
Plain $\mathrm{kV}^{\dagger}$ & $0.1-0.6$ & $0.1-3$ & Cyberknife, ExacTrac \\
Plain $\mathrm{MV}^{\dagger}$ & $1-10$ & $0.1-3$ & Various EPID and portal devices \\
$\mathrm{kV} \mathrm{CBCT}^{\ddagger}$ & $2-3$ & $2-4$ & ARTISTE, OBI, XVI \\
$\mathrm{MV} \mathrm{CBCT}_{\mathrm{kV} \mathrm{FBCT}}$ & $5-15$ & $2-3$ & MVision \\
MV FBCT & $0.8-2.8$ & 15 & CTVision, EXaCT \\
\hline
\end{tabular}

FBCT: fan-beam computed tomography scanning.

* excludes image interpretation and action on observations.

\&BATCAM, Best nomos, Pittsburgh, PA; Clarity and XVI, Elekta, Stockholm, Sweden; Cyberknife and Tomotherapy, Accuray, Sunnyvale, CA; EXaCT, ExacTrac, and OBI, Varian Medical Systems, Inc., Palo Alto, CA; ARTISTE, CTVision, and MVision, Siemens AG, Erlangen, Germany.

${ }^{\dagger}$ For 2 incidences.

${ }^{\ddagger}$ Full soft-tissue scan, $360^{\circ}$.

${ }^{\S}$ involves couch rotation and CT translation because CT scanning is not on linac gantry.

(ii) PTV Margins. One challenge facing RT is organ motion. This is especially relevant for prostate cancer. Recent studies have shown a significant degree of prostate motion, both inter- and intrafraction, and its adverse effects on IMRT dosimetry [23-25]. Appropriate margins have to be applied to ensure the targets receiving desired prescription dose. The magnitude of margin depends on the delivery technique. Various strategies have been studied, such as implanting fiducial gold seed markers (FMs) into the prostate [26-29], implanting beacon transponders into the prostate that allows for continuous, real-time localization [30-32], using an endorectal balloon to limit prostate motion during treatment [33], verifying the prostate position using B-mode acquisition, and targeting transabdominal ultrasonography before each treatment [34-37] or helical tomotherapy [24, 38, 39] or with CBCT [40-42]. Collectively, these strategies represent different attempts at IGRT, ultimately permitting the reduction of the PTV margins because of improved setup accuracy and reproducibility. Only with reduced margin, IMRT planning can take full advantage of image guidance and generate plans with reduced normal organ dose. Otherwise, the treatment plan will be identical with or without image guidance. In our study, similar PTV margins were used in both groups.

Fiducial markers can facilitate the localization of prostate. Soft-tissue targeting (prostate) requires a higher-dose imaging technique, but high-contrast targets, such as bone or metallic fiducial markers, can be accurately visualized at imaging doses as low as 0.1 to $0.5 \mathrm{cGy}$. Different registration can result from which landmarks are used as the reference, either fiducial markers, soft-tissue registration, or bony anatomy structures. Alander et al. [43] investigated the prostate bed localization difference between soft-tissue registration and gold seed fiducial localization. They found that the gold seed fiducial localization is better than bone localization and soft-tissue registration in daily IGRT. The CBCT and the bony structure alignment can reduce setup errors as compared to the localization with skin marks. Ost et al. [44] used anterior rectal wall to match posterior wall of the prostate bed of the CBCT with the planning CT and the registration was performed by a radiation oncologist and a therapist. In our study, no fiducial markers were used. CBCT for first 2-3 treatment fractions and last five fractions were supervised by a radiation oncologist. Radiation therapists did the rest of registration sessions by bony alignment. If we had implanted fiducial markers, therapists alone would be able to perform the registration more consistently. This was the reason we kept the PTV margins similar between the two groups. Without margin reduction, the advantage of image guidance was not fully realized.

(iii) Prescription Dose. Total radiation dose delivered to the target was one of the most important predictors of long-term biochemical tumor control [45]. Published randomized trials [46-49] demonstrated significant improvements in biochemical tumor control for low-risk patients and intermediate- and high-risk patients with escalated dose. It is reasonable to assume that additional escalation of the radiation dose for intermediate-risk patients and, especially, high-risk patients would be associated with further improvements in tumorcontrol outcomes. Zelefsky et al. [45] reported 2551 patients with clinical stages T1-T3 prostate cancer. Dose levels of $\geq 75.6 \mathrm{~Gy}$ for low-risk patients were associated with improved long-term PSA-RFS outcomes, and for higher-risk patients they observed improved biochemical control with $\geq 81 \mathrm{~Gy}$. In our study, the PTV dose of two groups was the same (76$80 \mathrm{~Gy})$. So it was not a surprise to see that the clinical tumorcontrol outcome was similar between IG-IMRT and IMRT groups.

(iv) Sample Size and Follow-Up Length. As shown in Table 7, the percentage of grade 2 acute GI and GU toxicity was 17\% and $42 \%$ in six previously reported studies. They were slightly higher than our IMRT and IG-IMRT groups. Statistically, there were no differences between our two groups due to our small sample sizes and the low probability of side effects. The incidence of grade 2 and higher late rectal and bladder toxicity was so low for both treatment groups $(1.5 \%$ and $3 \%$, resp.; $P=0.41$ ) that only 1 or 2 patients had any problems. 
TABLE 7: Acute gastrointestinal (GI) and genitourinary (GU) toxicity in the literature on radiation therapy of prostate cancer.

\begin{tabular}{lccccccccc}
\hline Study & $\begin{array}{c}\text { Patients } \\
(n)\end{array}$ & $\begin{array}{c}\text { Total dose } \\
(\text { Gy })\end{array}$ & $\begin{array}{c}\text { Fractions } \\
(n)\end{array}$ & \multicolumn{2}{c}{ EQD2 } & IGRT & \multicolumn{2}{c}{ Acute GI (\%) } & \multicolumn{2}{c}{ Acute GU (\%) } \\
Grade 2 & Grade 3 & Grade 2 & Grade 3 \\
\hline Pollack et al. 2006 [7] & 100 & 76 (D95) & 38 & 76 & Daily & 8 & 0 & 54 \\
& & 70.4 (D95) & 28 & 84.6 & & 18 & 0 & 40 & 8 \\
Fonteyne et al. 2008 [8] & 230 & 78 (median) & 38 & 79.1 & Daily & 11 & 0 & 41 & 7 \\
Ghadjar et al. 2008 [54] & 39 & 80 (median) & 40 & 80 & Daily & 3 & 0 & 56 & 8 \\
Lips et al. 2008 [9] & 331 & 76 (mean) & 35 & 79.7 & Daily & 30 & 0 & 47 & 3 \\
Marchand et al. 2010 [55] & 55 & 72.2 & 38 & 70.1 & Daily & 12.7 & 0 & 38.2 & 1.8 \\
Deville et al. 2010 [56] & 30 & 79.2 & 44 & 74.7 & Daily & 13 & 0 & 50 & 0 \\
Our own data & 127 & 78.6 (mean) & 39 & 78.9 & Customized & 4 & 0 & 17.3 & 0 \\
\hline
\end{tabular}

More patients would be required to demonstrate any difference in the tolerance profile of treated patients. In addition, the follow-up length in our study was relatively short with only 23 months (median) in the IG-IMRT group. Longer followup can potentially magnify the differences between the groups.

In conclusion, our study did not show significant improvement in biochemical control at 5 years in the low-, intermediate-, and high-risk prostate patients with IG-IMRT compared with IMRT alone; neither did we observe any statistical difference in grade 2 and higher acute GI and $\mathrm{GU}$ toxicity rates. We believe it was due to multiple factors including the frequency of image guidance, PTV margin and dose, sample size, and length of followup. In order to maximize the benefit of IGRT, those parameters should be carefully evaluated.

\section{Conflict of Interests}

The authors declare that there is no conflict of interests regarding the publication of this paper.

\section{Acknowledgment}

This work was supported by the National Natural Science Foundation of China (Grant nos. 81101694 and 81372415).

\section{References}

[1] M. J. Zelefsky, Z. Fuks, L. Happersett et al., "Clinical experience with intensity modulated radiation therapy (IMRT) in prostate cancer," Radiotherapy \& Oncology, vol. 55, no. 3, pp. 241-249, 2000.

[2] J. M. Michalski, Y. Yan, D. Watkins-Bruner et al., "Preliminary toxicity analysis of 3-dimensional conformal radiation therapy versus intensity modulated radiation therapy on the high-dose arm of the radiation therapy oncology group 0126 prostate cancer trial," International Journal of Radiation Oncology, Biology, Physics, vol. 87, no. 5, pp. 932-938, 2013.

[3] B. S. Teh, W. Mai, W. H. Grant III et al., "Intensity modulated radiotherapy (IMRT) decreases treatment-related morbidity and potentially enhances tumor control," Cancer Investigation, vol. 20 , no. 4, pp. 437-451, 2002.
[4] B. S. Teh, W. Mai, B. M. Uhl et al., "Intensity-modulated radiation therapy (IMRT) for prostate cancer with the use of a rectal balloon for prostate immobilization: acute toxicity and dose-volume analysis," International Journal of Radiation Oncology Biology Physics, vol. 49, no. 3, pp. 705-712, 2001.

[5] M. Roach III, M. DeSilvio, C. Lawton et al., "Phase III trial comparing whole-pelvic versus prostate-only radiotherapy and neoadjuvant versus adjuvant combined androgen suppression: Radiation Therapy Oncology Group 9413," Journal of Clinical Oncology, vol. 21, no. 10, pp. 1904-1911, 2003.

[6] A. Wang-Chesebro, P. Xia, J. Coleman, C. Akazawa, and M. Roach III, "Intensity-modulated radiotherapy improves lymph node coverage and dose to critical structures compared with three-dimensional conformal radiation therapy in clinically localized prostate cancer," International Journal of Radiation Oncology, Biology, Physics, vol. 66, no. 3, pp. 654-662, 2006.

[7] A. Pollack, A. L. Hanlon, E. M. Horwitz et al., "Dosimetry and preliminary acute toxicity in the first 100 men treated for prostate cancer on a randomized hypofractionation dose escalation trial," International Journal of Radiation Oncology Biology Physics, vol. 64, no. 2, pp. 518-526, 2006.

[8] V. Fonteyne, G. Villeirs, B. Speleers et al., "Intensity-modulated radiotherapy as primary therapy for prostate cancer: report on acute toxicity after dose escalation with simultaneous integrated boost to intraprostatic lesion," International Journal of Radiation Oncology Biology Physics, vol. 72, no. 3, pp. 799-807, 2008.

[9] I. M. Lips, H. Dehnad, C. H. van Gils, A. E. B. Kruger, U. A. van der Heide, and M. van Vulpen, "High-dose intensitymodulated radiotherapy for prostate cancer using daily fiducial marker-based position verification: acute and late toxicity in 331 patients," Radiation Oncology, vol. 3, no. 1, article 15, 2008.

[10] H. T. Chung, P. Xia, L. W. Chan, E. Park-Somers, and M. Roach III, "Does image-guided radiotherapy improve toxicity profile in whole pelvic-treated high-risk prostate cancer? comparison between IG-IMRT and IMRT,' International Journal of Radiation Oncology Biology Physics, vol. 73, no. 1, pp. 53-60, 2009.

[11] R. de Crevoisier, P. Pommier, J. Bachaud et al., "Image-guided radiation therapy (IGRT) in prostate cancer: preliminary results in prostate registration and acute toxicity of a randomized study," International Journal of Radiation Oncology, Biology, Physics, vol. 75, no. 3, p. s99, 2009.

[12] M. J. Zelefsky, M. Kollmeier, B. Cox et al., "Improved clinical outcomes with high-dose image guided radiotherapy compared with non-IGRT for the treatment of clinically localized prostate cancer," International Journal of Radiation Oncology Biology Physics, vol. 84, no. 1, pp. 125-129, 2012. 
[13] D. E. Spratt, X. Pei, J. Yamada, M. A. Kollmeier, B. Cox, and M. J. Zelefsky, "Long-term survival and toxicity in patients treated with high-dose intensity modulated radiation therapy for localized prostate cancer," International Journal of Radiation Oncology Biology Physics, vol. 85, no. 3, pp. 686-692, 2013.

[14] A. Bujold, T. Craig, D. Jaffray, and L. A. Dawson, "Image-guided radiotherapy: has it influenced patient outcomes?" Seminars in Radiation Oncology, vol. 22, no. 1, pp. 50-61, 2012.

[15] M. K. Islam, T. G. Purdie, B. D. Norrlinger et al., "Patient dose from kilovoltage cone beam computed tomography imaging in radiation therapy," Medical Physics, vol. 33, no. 6, pp. 1573-1582, 2006.

[16] C. Walter, J. Boda-Heggemann, H. Wertz et al., "Phantom and in-vivo measurements of dose exposure by image-guided radiotherapy (IGRT): MV portal images vs. $\mathrm{kV}$ portal images vs. cone-beam CT," Radiotherapy and Oncology, vol. 85, no. 3, pp. 418-423, 2007.

[17] G. X. Ding and C. W. Coffey, "Radiation dose from kilovoltage cone beam computed tomography in an image-guided radiotherapy procedure," International Journal of Radiation Oncology, Biology, Physics, vol. 73, no. 2, pp. 610-617, 2009.

[18] J. Simon, F. Eschwege, and L. El Hajj, “409 Patients over exposed during radiotherapy for prostate cancer after daily use of portal imaging controls," International Journal of Radiation Oncology, Biology, Physics, vol. 78, no. 3, p. S361, 2010.

[19] L. Perrier, M. Morelle, P. Pommier et al., "Cost of prostate image-guided radiation therapy: results of a randomized trial," Radiotherapy and Oncology, vol. 106, no. 1, pp. 50-58, 2013.

[20] J. C. Stroom, H. C. J. de Boer, H. Huizenga, and A. G. Visser, "Inclusion of geometrical uncertainties in radiotherapy treatment planning by means of coverage probability," International Journal of Radiation Oncology Biology Physics, vol. 43, no. 4, pp. 905-919, 1999.

[21] M. van Herk, P. Remeijer, C. Rasch, and J. V. Lebesque, "The probability of correct target dosage: dose-population histograms for deriving treatment margins in radiotherapy," International Journal of Radiation Oncology Biology Physics, vol. 47, no. 4, pp. 1121-1135, 2000.

[22] M. Van Herk, P. Remeijer, and J. V. Lebesque, "Inclusion of geometric uncertainties in treatment plan evaluation," International Journal of Radiation Oncology Biology Physics, vol. 52, no. 5, pp. 1407-1422, 2002.

[23] N. P. Orton and W. A. Tomé, "The impact of daily shifts on prostate IMRT dose distributions," Medical Physics, vol. 31, no. 10, pp. 2845-2848, 2004.

[24] P. A. Kupelian, K. M. Langen, O. A. Zeidan et al., "Daily variations in delivered doses in patients treated with radiotherapy for localized prostate cancer," International Journal of Radiation Oncology Biology Physics, vol. 66, no. 3, pp. 876-882, 2006.

[25] V. Landoni, B. Saracino, S. Marzi et al., "A study of the effect of setup errors and organ motion on prostate cancer treatment with IMRT,' International Journal of Radiation Oncology, Biology, Physics, vol. 65, no. 2, pp. 587-594, 2006.

[26] J. M. Schallenkamp, M. G. Herman, J. J. Kruse, and T. M. Pisansky, "Prostate position relative to pelvic bony anatomy based on intraprostatic gold markers and electronic portal imaging," International Journal of Radiation Oncology Biology Physics, vol. 63, no. 3, pp. 800-811, 2005.

[27] L. E. Millender, M. Aubin, J. Pouliot, K. Shinohara, and M. Roach III, "Daily electronic portal imaging for morbidly obese men undergoing radiotherapy for localized prostate cancer,"
International Journal of Radiation Oncology Biology Physics, vol. 59, no. 1, pp. 6-10, 2004.

[28] M. G. Herman, T. M. Pisansky, J. J. Kruse, J. I. Prisciandaro, B. J. Davis, and B. F. King, "Technical aspects of daily online positioning of the prostate for three-dimensional conformal radiotherapy using an electronic portal imaging device," International Journal of Radiation Oncology Biology Physics, vol. 57, no. 4, pp. 1131-1140, 2003.

[29] E. Vigneault, J. Pouliot, J. Laverdière, R. Jean, and M. Dorion, "Electronic portal imaging device detection of radioopaque markers for the evaluation of prostate position during megavoltage irradiation: a clinical study," International Journal of Radiation Oncology Biology Physics, vol. 37, no. 1, pp. 205-212, 1997.

[30] T. R. Willoughby, P. A. Kupelian, J. Pouliot et al., "Target localization and real-time tracking using the Calypso 4D localization system in patients with localized prostate cancer," International Journal of Radiation Oncology Biology Physics, vol. 65, no. 2, pp. 528-534, 2006.

[31] P. Kupelian, T. Willoughby, A. Mahadevan et al., "Multiinstitutional clinical experience with the Calypso System in localization and continuous, real-time monitoring of the prostate gland during external radiotherapy," International Journal of Radiation Oncology Biology Physics, vol. 67, no. 4, pp. 10881098, 2007.

[32] D. W. Litzenberg, J. M. Balter, S. W. Hadley et al., "Influence of intrafraction motion on margins for prostate radiotherapy," International Journal of Radiation Oncology Biology Physics, vol. 65 , no. 2, pp. 548-553, 2006.

[33] E. N. J. T. Van Lin, L. P. Van Der Vight, J. A. Witjes, H. J. Huisman, J. W. Leer, and A. G. Visser, "The effect of an endorectal balloon and off-line correction on the interfraction systematic and random prostate position variations: a comparative study," International Journal of Radiation Oncology Biology Physics, vol. 61, no. 1, pp. 278-288, 2005.

[34] J. Lattanzi, S. McNeeley, W. Pinover et al., "A comparison of daily CT localization to a daily ultrasound-based system in prostate cancer," International Journal of Radiation Oncology, Biology, Physics, vol. 43, no. 4, pp. 719-725, 1999.

[35] C. F. Serago, S. J. Chungbin, S. J. Buskirk, G. A. Ezzell, A. C. Collie, and S. A. Vora, "Initial experience with ultrasound localization for positioning prostate cancer patients for external beam radiotherapy," International Journal of Radiation Oncology Biology Physics, vol. 53, no. 5, pp. 1130-1138, 2002.

[36] E. Huang, L. Dong, A. Chandra et al., "Intrafraction prostate motion during IMRT for prostate cancer," International Journal of Radiation Oncology Biology Physics, vol. 53, no. 2, pp. 261-268, 2002.

[37] T. J. Scarbrough, N. M. Golden, J. Y. Ting et al., "Comparison of ultrasound and implanted seed marker prostate localization methods: Implications for image-guided radiotherapy," International Journal of Radiation Oncology Biology Physics, vol. 65, no. 2, pp. 378-387, 2006.

[38] P. A. Kupelian, C. Lee, K. M. Langen et al., "Evaluation of image guidance strategies in the treatment of localized prostate cancer," International Journal of Radiation Oncology Biology Physics, vol. 70, no. 4, pp. 1151-1157, 2008.

[39] D. M. Drabik, M. A. MacKenzie, and G. B. Fallone, "Quantifying appropriate PTV setup margins: analysis of patient setup fidelity and intrafraction motion using post-treatment megavoltage 
computed tomography scans," International Journal of Radiation Oncology, Biology, Physics, vol. 68, no. 4, pp. 1222-1228, 2007.

[40] D. J. Moseley, E. A. White, K. L. Wiltshire et al., "Comparison of localization performance with implanted fiducial markers and cone-beam computed tomography for on-line image-guided radiotherapy of the prostate," International Journal of Radiation Oncology Biology Physics, vol. 67, no. 3, pp. 942-953, 2007.

[41] J. Nijkamp, F. J. Pos, T. T. Nuver et al., "Adaptive radiotherapy for prostate cancer using kilovoltage cone-beam computed tomography: first clinical results," International Journal of Radiation Oncology Biology Physics, vol. 70, no. 1, pp. 75-82, 2008.

[42] M. H. P. Smitsmans, J. De Bois, J. Sonke et al., "Automatic prostate localization on cone-beam CT scans for high precision image-guided radiotherapy," International Journal of Radiation Oncology Biology Physics, vol. 63, no. 4, pp. 975-984, 2005.

[43] E. Alander, H. Visapaa, M. Kouri et al., "Gold seed fiducials in analysis of linear and rotational displacement of the prostate bed," Radiotherapy and Oncology, vol. 110, no. 2, pp. 256-260, 2014.

[44] P. Ost, G. de Meerleer, W. de Gersem, A. Impens, and W. de Neve, "Analysis of prostate bed motion using daily cone-beam computed tomography during postprostatectomy radiotherapy," International Journal of Radiation Oncology Biology Physics, vol. 79, no. 1, pp. 188-194, 2011.

[45] M. J. Zelefsky, X. Pei, J. F. Chou et al., "Dose escalation for prostate cancer radiotherapy: predictors of long-term biochemical tumor control and distant metastases-free survival outcomes," European Urology, vol. 60, no. 6, pp. 1133-1139, 2011.

[46] A. Pollack, G. K. Zagars, G. Starkschall et al., "Prostate cancer radiation dose response: results of the M. D. Anderson phase III randomized trial," International Journal of Radiation Oncology Biology Physics, vol. 53, no. 5, pp. 1097-1105, 2002.

[47] A. L. Zietman, K. Bae, J. D. Slater et al., "Randomized trial comparing conventional-dose with high-dose conformal radiation therapy in early-stage adenocarcinoma of the prostate: longterm results from Proton Radiation Oncology Group/American College Of Radiology 95-09," Journal of Clinical Oncology, vol. 28, no. 7, pp. 1106-1111, 2010.

[48] S. T. Peeters, W. D. Heemsbergen, P. C. Koper et al., "Doseresponse in radiotherapy for localized prostate cancer: results of the Dutch multicenter randomized phase III trial comparing 68 Gy of radiotherapy with 78 Gy," Journal of Clinical Oncology, vol. 24, no. 13, pp. 1990-1996, 2006.

[49] D. P. Dearnaley, M. R. Sydes, J. D. Graham et al., "Escalateddose versus standard-dose conformal radiotherapy in prostate cancer: first results from the MRC RT01 randomised controlled trial," Lancet Oncology, vol. 8, no. 6, pp. 475-487, 2007.

[50] M. J. Murphy, J. Balter, S. Balter et al., "The management of imaging dose during image-guided radiotherapy: report of the AAPM Task Group 75," Medical Physics, vol. 34, no. 10, pp. 40414063, 2007.

[51] G. Delpon, C. Llagostera, M. Le Blanc et al., "Use of IGRT for prostate cancers (OBI-CBCT VarianŮ, ExacTrac $®$ BrainLABU゚ and MVCT TomoTherapy)," Cancer/Radiotherapie, vol. 13, no. 5, pp. 399-407, 2009.

[52] C. Peng, K. Kainz, C. Lawton, and X. A. Li, "A Comparison of daily megavoltage $\mathrm{CT}$ and ultrasound image guided radiation therapy for prostate cancer," Medical Physics, vol. 35, no. 12, pp. 5619-5628, 2008.

[53] C. W. Cheng, J. Wong, L. Grimm, M. Chow, M. Uematsu, and A. Fung, "Commissioning and clinical implementation of a sliding gantry CT scanner installed in an existing treatment room and early clinical experience for precise tumor localization," The American Journal of Clinical Oncology, vol. 26, no. 3, pp. e28e36, 2003.

[54] P. Ghadjar, J. Vock, D. Vetterli et al., "Acute and late toxicity in prostate cancer patients treated by dose escalated intensity modulated radiation therapy and organ tracking," Radiation Oncology, vol. 3, no. 1, article 35, 2008.

[55] V. Marchand, S. Bourdin, C. Charbonnel et al., "No impairment of quality of life 18 months after high-dose intensity-modulated radiotherapy for localized prostate cancer: a prospective study," International Journal of Radiation Oncology Biology Physics, vol. 77, no. 4, pp. 1053-1059, 2010.

[56] C. Deville, S. Both, W.-T. Hwang, Z. Tochner, and N. Vapiwala, "Clinical toxicities and dosimetric parameters after wholepelvis versus prostate-only intensity-modulated radiation therapy for prostate cancer," International Journal of Radiation Oncology, Biology, Physics, vol. 78, no. 3, pp. 763-772, 2010. 


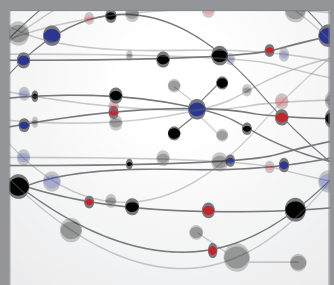

The Scientific World Journal
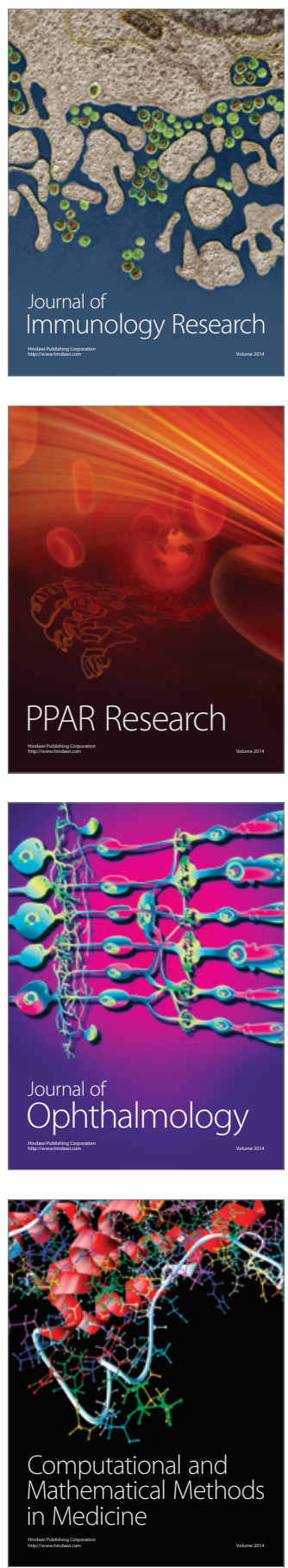

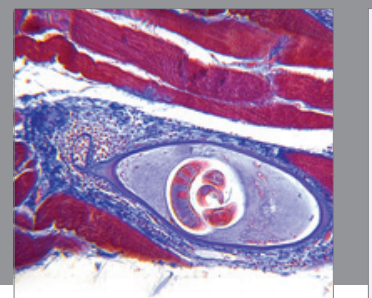

Gastroenterology

Research and Practice
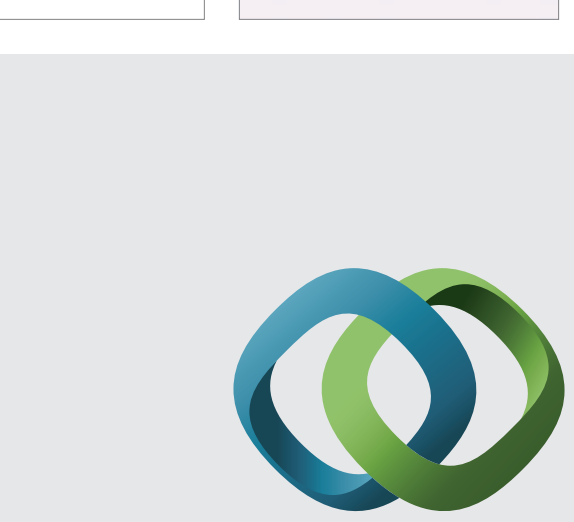

\section{Hindawi}

Submit your manuscripts at

http://www.hindawi.com
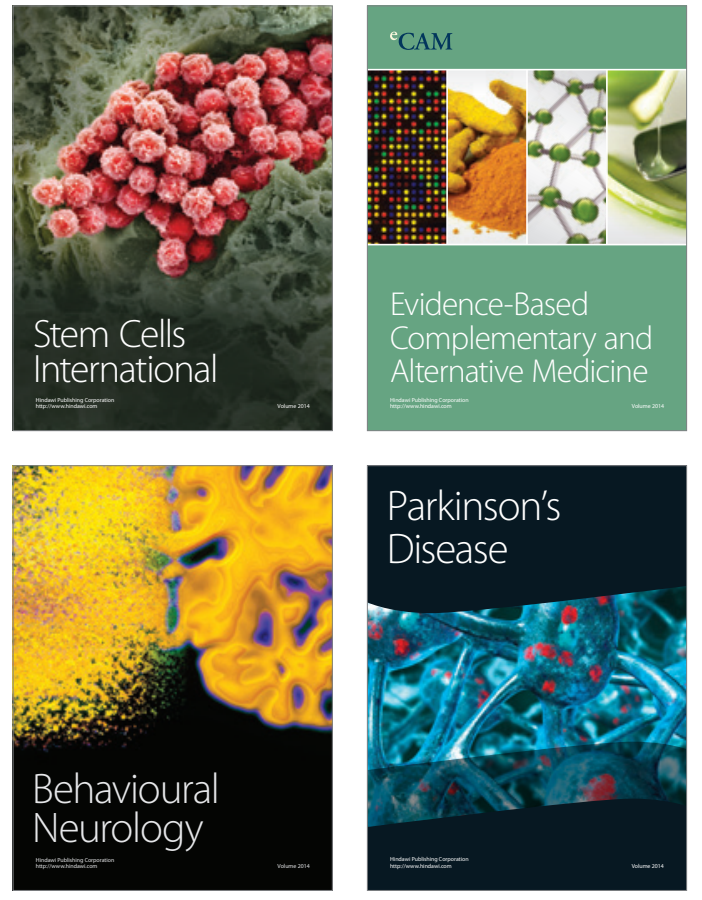
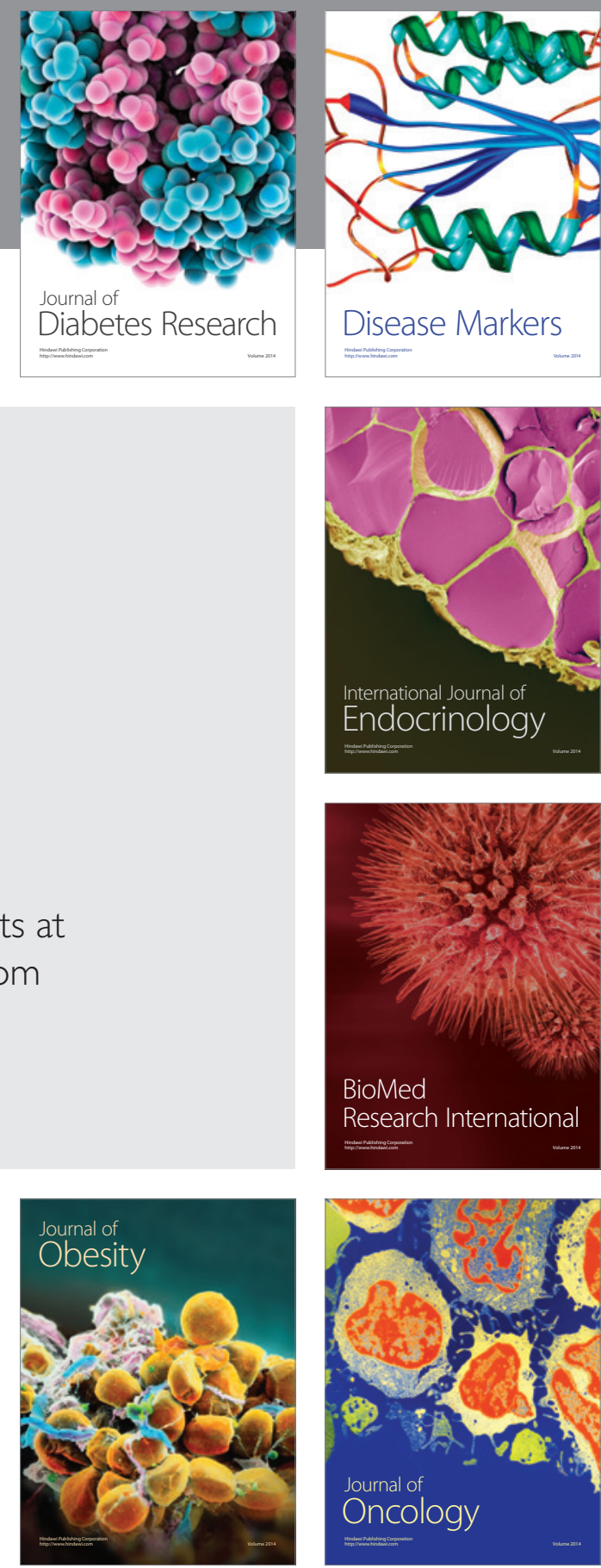

Disease Markers
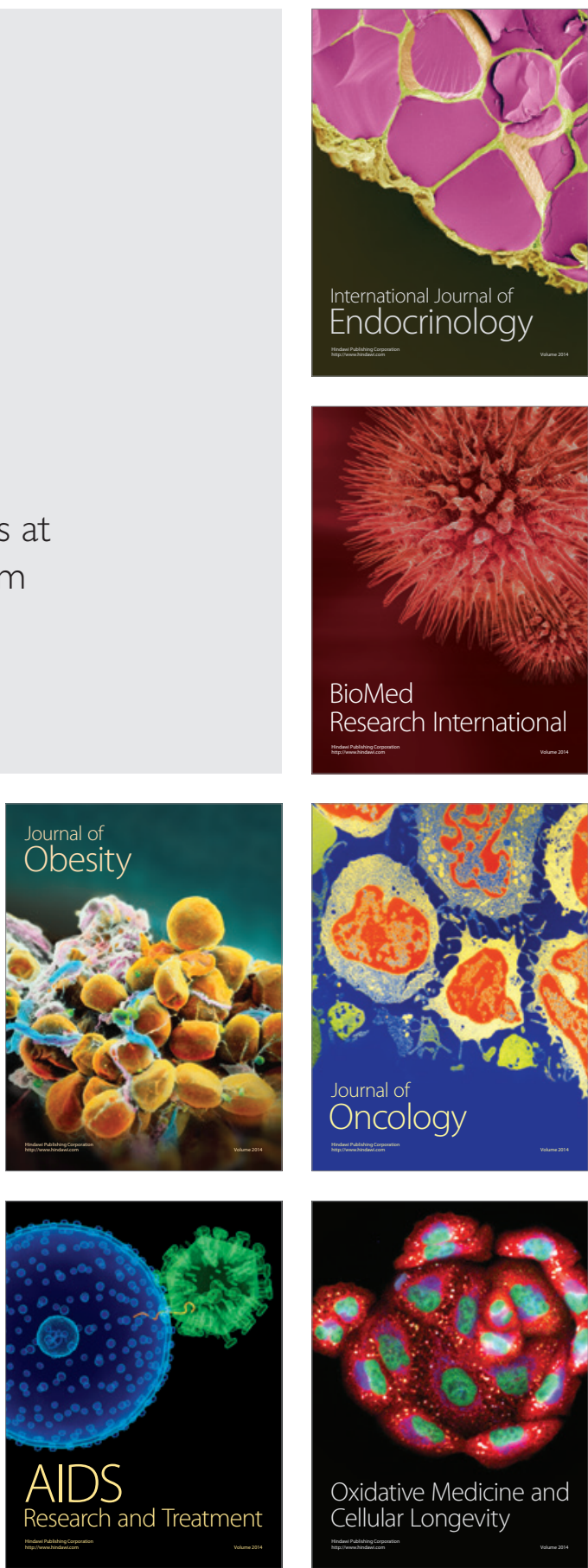